\title{
STEADY STATE ANALYSIS OF PHOTON AVALANCHE EFFECT
}

\author{
F. Pellé and P. Goldner \\ Groupe d'Optique des Terres Rares \\ Laboratoire de Physico-Chimie des Matériaux-UPR 211 CNRS \\ 1 pl. Aristide Briand, Meudon Bellevue, France \\ France-Telecom, CNET Laboratoire de Bagneux, B.P.107, 92225 Bagneux, France
}

An analysis of the three-level model for avalanche up-conversion in a steady state case is presented. Until now, no quantitative criterion has been defined to distinguish a valanche from nonlinear processes giving rise to anti-Stokes emissions. From our model, we drive a quantitative limit for the ratio between non resonant and resonant absorption cross-section to observe an avalanche process. Application of these calculations to practical cases demonstrates the ability of our model to predict an avalanche behavior. The interest of avalanche to pump up-conversion lasers is discussed by introducing a term in the rate equations due to the stimulated emission.

PACS numbers: 42.50.IIz, 42.62.Fi, 42.70. $\mathrm{Fj}, 78.50 . \mathrm{Ec}$

\section{Introduction}

Up-conversion luminescence is easily obtained in rare earth doped materials. Several nonlinear processes can be responsible for these anti-Stokes emissions and their quantum efficiency is several order of magnitude higher than the second harmonic generation one [1]. Therefore, rare earth doped materials represent a good opportunity for compact diode pumped solid state laser sources especially for the blue-green emission. Among different energy transfer schemes giving rise to up-conversion, avalanche pumping has been recently proposed to obtain an efficient visible laser emission. First observed by Chivian et al. [2] with $\mathrm{Pr}^{3+}$ in $\mathrm{LaCl}_{3}$, the photon avalanche effect is characterized by an excitation resonant only with a transition between excited states, a pump threshold above which up-converted fluorescence intensity increases substantially and a built-up time of the emission much longer than the decay times of the involved electronic levels. These particular behaviors will be detailed by F. Auzel in this conference. Several papers are devoted to the study of the avalanche effect [3-9] and models including three or four levels and based on rate equations have been proposed and analyzed using several methods [4,6,9-12]. However until now, no quantitative and meaningful limit between avalanche and non-avalanche behavior has been defined. We present 
an analysis of the three-level model for the avalanche effect in a steady state case. We propose a quantitative criterion to distinguish avalanche from other nonlinear processes such as addition of photons by energy transfer, excited state absorption, etc. The calculations will be applied to $\mathrm{Er}^{3+}$ and $\mathrm{Nd}^{3+}$ (well described by a three-level system) in $\mathrm{LiYF}_{4}$ and show that our model can be used to predict an avalanche behavior providing that the electronic absorption cross-sections and the Stokes and anti-Stokes multiphonon assisted probabilities are known.

An application to the avalanche pumped up-conversion lasers needs to introduce an additional term in the rate equations due to the stimulated emission. The efficiency of avalanche pumping will be compared to the resonant excitation case for $\mathrm{Er}^{3+}$ and $\mathrm{Nd}^{3+}$ in $\mathrm{LiYF}_{4}$.

\section{Analysis of avalanche up-conversion}

\subsection{The three-level model}

In usual doped materials, where most of the doping ions are randomly distributed, a fast diffusion between impurities is likely to occur so in this case the system can be modeled by rate equations [13]. A fast diffusion assumption is justified since it has been found to be of great importance in the case of a valanche [14]. In this model, we neglect short lived levels. The relaxations and energy transfer connecting the three levels are represented in Fig. 1. In the steady state, the corresponding rate equations are $[10,12]$

$$
\begin{aligned}
& \dot{p}_{1}=-W_{1} p_{1}-\alpha p_{1} p_{3}+r_{2} p_{2}+r_{31} p_{1}=0, \\
& \dot{p}_{2}=-\left(W_{2}+r_{2}\right) p_{2}+r_{32} p_{3}+W_{1} p_{1}+2 \alpha p_{1} p_{3}=0, \\
& \dot{p}_{3}=-\left(r_{32}+r_{31}\right) p_{3}-\alpha p_{1} p_{3}+W_{2} p_{2}=0, \\
& \sum_{i} p_{i}=1,
\end{aligned}
$$

where $W_{1}$ and $W_{2}$ are the pumping rates from level 1 to level 2 and from level 2 to level 3 , respectively, $r_{i j}$ is the total relaxation rate from level $i$ to level $j$ for

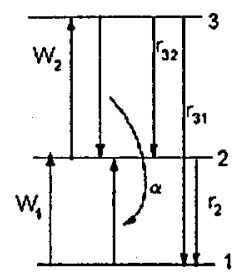

Fig. 1. Relaxations and energy transfer involved in avalanche and CRESA processes for a three-level system. 
the isolated ion and $\alpha$ is the cross-relaxation rate connecting levels 1 and 3 to the intermediate one. $p_{i}$ is the level $i$ occupation probability. Neglecting short lived levels, this model applies to $\mathrm{Nd}^{3+}$ (pumped at $603.6 \mathrm{~nm}$ ) [4], $\mathrm{Er}^{3+}$ (pumped at $578 \mathrm{~nm}$ ) [8] and $\mathrm{Tm}^{3+}$ ions (pumped at $1.04 \mu \mathrm{m}$ ) [12].

We will particularly focused our interest to the dependence on the upper level population $p_{3}^{\beta}$ with respect to the pumping rate $W_{2}$ and the ratio $\beta=W_{1} / W_{2}$. Since the avalanche effect is usually observed under one pumping source, the last parameter is constant and equal to the ratio between the $1 \rightarrow 2$ and $2 \rightarrow 3$ effective absorption cross-sections.

\subsection{Influence of the ground state absorption on upper level population}

In Fig. 2, the $p_{3}^{\beta}$ population is plotted against the $W_{2}$ pumping rate, the parameters used for the computations are those of $\mathrm{Nd}^{3+}$ in $\mathrm{LiYF}_{4}\left(r_{2}=1905 \mathrm{~s}^{-1}\right.$, $r_{32}=16000 \mathrm{~s}^{-1}, r_{31}=16000 \mathrm{~s}^{-1}, \alpha=15600 \mathrm{~s}^{-1}$ ) [10]. The ratio $\beta$ varies from 0 to 1 . The $\beta=0$ "ideal case" (zero-ground state absorption (zero-GSA)) has no physical meaning in the case of RE-doped materials since residual absorption (around $10^{-6}$ ) always occurs even for an excitation several thousands of wave numbers far away from resonance $[8,15]$. The case $\beta=1$ corresponds to the fully resonant case. As shown in Fig. 2, no discontinuity is observed for $p_{3}^{\beta}\left(W_{2}\right)$ while increasing $\beta$. For very small values of $\beta\left(\leq 10^{-3}\right), p_{3}^{\beta}$ approaches the solution obtained in the ideal case. Ilowever the threshold power is no more precisely defined. For higher values of $\beta$, the curves smoothly vary and have little in common with the zero-GSA. In this case, the behavior of $p_{3}^{\beta}$ does not exhibit any avalanche effect. From this simulation, the importance of the ground state absorption to obtain a true avalanche process is highlighted. It clearly appears that avalanche will only occur for very small values of $\beta$, i.e. a large detuning between excitation and resonant absorption from the ground state.

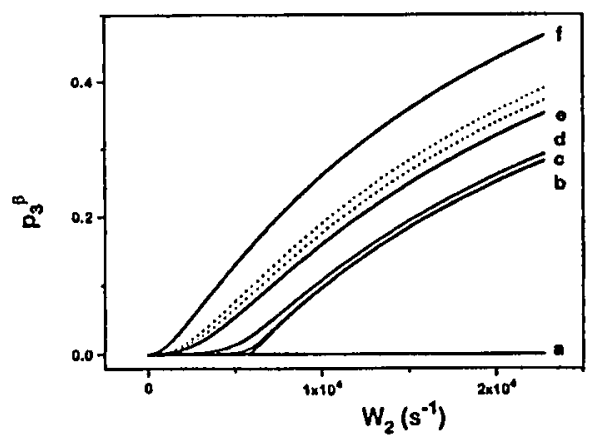

Fig. 2. Upper level population dependence on $W_{2}$ pumping rate for various values of $\beta$ (other parameters correspond to $\left.\mathrm{Nd}^{3+}: \mathrm{YLiF}_{4}\right) . \beta=0(\mathrm{a}, \mathrm{b}), 10^{-3}(\mathrm{c}), 10^{-2}(\mathrm{~d}), 10^{-1}$ (e), 1 (f).The dotted lines represent the avalanche limits in Refs. [16] and [10]. On this scale, the curves $\beta=0$ and $\beta=1.7 \times 10^{-4}$ cannot be separated. 


\subsection{Analysis of the rate equations}

In this section, the solutions of Eq. (1) approaching the zero-GSA will be discussed and conditions on $\beta$ to observe an avalanche effect will be derived. We first briefly discuss the ideal case for clarity.

\subsubsection{Zero-GSA $\left(W_{1}=0\right)$}

Up-conversion from level 3 will be observed only if $\alpha>r_{31}$ and a steady state population in level 3 will be established above a critical value of the pumping rate $\left(W_{\mathrm{c}}\right)$ given by

$$
W_{\mathrm{c}}=\frac{\left(\alpha+r_{31}+r_{32}\right) r_{2}}{\alpha-r_{31}} \text {. }
$$

Above this value, $p_{3}^{0}$ is 0 or equal to

$$
p_{3}^{0}\left(W_{2}\right)=\frac{\left(\alpha-r_{31}\right)\left(W_{2}-W_{c}\right)}{\alpha\left(2 r_{31}+r_{32}-r_{2}+W_{2}\right)} .
$$

Among the terms which produce a loss of the upper level population, $r_{31}$ is the most important one because $r_{32}$ can always be compensated by increasing the $W_{2}$ pumping rate. The $r_{2}$ relaxation rate reduces the $2 \rightarrow 3$ pumping efficiency but has only an effect on the $W_{c}$ threshold value.

In conclusion, a low threshold and fast increases of the up-converted emission require a metastable intermediate state and a strong $[3,1] \rightarrow[2,2]$ cross-relaxation.

\subsubsection{General case $\left(W_{1} \neq 0\right)$}

The general solution of Eq. (1) is given by

$$
\begin{aligned}
& p_{3}^{\beta}\left(W_{2}\right)=\frac{-b+\left(b^{2}-4 a c\right)^{1 / 2}}{2 a}, \\
& \text { where }\left\{\begin{array}{l}
a=\alpha\left(2 r_{31}+r_{32}-r_{2}+W_{2}\right), \\
b=\left(r_{31}-\alpha\right) W_{2}+\left(r_{31}+r_{32}\right) \beta W_{2}+r_{2}\left(\alpha+r_{31}+r_{32}\right)+\beta W_{2}^{2}, \\
c=-\beta W_{2}^{2} .
\end{array}\right.
\end{aligned}
$$

In this approach, to define a limit for $\beta$ which can be a criterion for avalanche behavior, we are mainly interested by the curves very close to the zero-GSA case. In this scheme we can simply use a zero order approximation for $p_{3}^{\beta}$ and keep $W_{\mathrm{c}}$ as the threshold pump power even if $\beta \neq 0$. Limits on $\beta$ for the avalanche up-conversion already given are too large since the corresponding curves (dashed lines in Fig. 2) are very far from the ideal case curve and clearly do not reveal any avalanche behavior. Restricting pumping rates close to $W_{\mathrm{c}}$ and $\beta$ to very small values, it can be shown from $p_{3}^{0}$ that the largest difference between $p_{3}^{\beta}$ and $p_{3}^{0}$ is precisely found for $W_{2}=W_{\mathrm{c}}$. A first order expression of $p_{3}^{\beta}$ at this pumping rate is given by

$$
p_{3}^{\beta}\left(W_{\mathrm{c}}\right) \approx \frac{W_{\mathrm{c}}}{\sqrt{\alpha\left(2 r_{31}+r_{32}-r_{2}+W_{\mathrm{c}}\right)}} \sqrt{\beta} .
$$

To determine a limit, it is necessary to scale properly $p_{3}^{\beta}$ because at the threshold $p_{3}^{0}=0$. We choose to scale $p_{3}^{\beta}$ between $\beta=0$ and $\beta=1$, so avalanche 
will be determined by the ratio $\left(p_{3}^{\beta}-p_{3}^{0}\right) / p_{3}^{1}\left(W_{\mathrm{c}}\right)=p_{3}^{\beta} / p_{3}^{1}\left(W_{\mathrm{c}}\right)$ computed at $W_{2}=W_{\mathrm{c}}$. The values of $R^{\beta}\left(W_{2}\right)$, for $W_{2}$ close to $W_{\mathrm{c}}$, using parameters of $\mathrm{Nd}^{3+}$ in $\mathrm{LiYF}_{4}$, are plotted in Fig. 3. From Eqs. (3) and (4), it can be shown that $\sqrt{\beta}<R^{\beta}\left(W_{c}\right)<2.5 \sqrt{\beta}$ for most sets of parameters. The limit to observe an avalanche behavior is then $R^{\beta}\left(W_{\mathrm{c}}\right)=10^{-2}$ and corresponds to a $\beta$ value well comprised between $10^{-5}$ and $10^{-4}$, domain, where the $p_{3}^{\beta}$ variations approach the ideal case. For higher values (i.e. moderate detuning), $p_{3}^{\beta}$ curves correspond to a cross-relaxation-enhanced-excited-state-absorption (CRESA). This kind of behavior has some similarities with the looping mechanism described by Brenier et al. $[16,17]$.

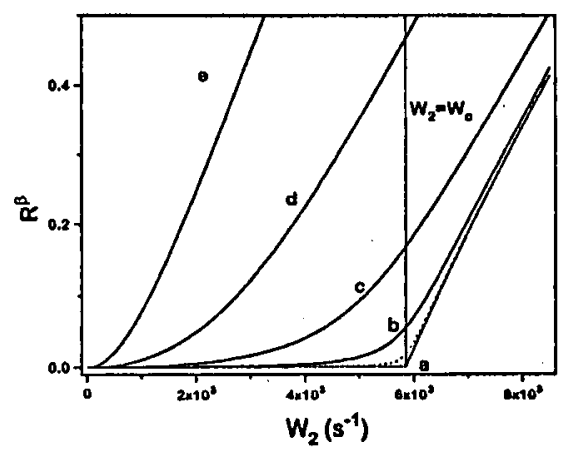

Fig. 3. $R$ parameter dependence on $W_{2}$ pumping rate. $\beta=0(\mathrm{a}), 10^{-3}(\mathrm{~b}), 10^{-2}$ (c), $10^{-1}(\mathrm{~d}), 1$ (e). The dashed line refers to the limit of avalanche behavior $\left(\beta=10^{-4}\right)$.

From a practical point of view, the $\beta$ parameter can be evaluated provided that $\alpha_{\mathrm{S}}$ and $\alpha_{\mathrm{AS}}$, cross-sections for Stokes and anti-Stokes multiphonon assisted absorption, respectively [15], are known.

\subsubsection{Comparison with experimental resulls}

We apply the above calculations to $\mathrm{Er}^{3+}$ and $\mathrm{Nd}^{3+}$ ions in $\mathrm{LiYF}_{4}$ for which all necessary quantities are a vailable. The $\alpha_{\mathrm{S}}$ and $\alpha_{\mathrm{AS}}$ (nearly independent of the $\mathrm{RE})$ have been measured at $300 \mathrm{~K}[8]$, they are found to be $9.2 \times 10^{-3} \mathrm{~cm}$ and $14.5 \times 10^{-3} \mathrm{~cm}$ respectively. From these values, it appears that the pump beam should be at least $1000 \mathrm{~cm}^{-1}$ (respectively $600 \mathrm{~cm}^{-1}$ ) away from the electronic levels for Stokes (respectively anti-Stokes) processes.

In the case of $\mathrm{Er}^{3+}$ in $\mathrm{LiYF}_{4}$ pumped at $578 \mathrm{~nm}$, the detuning of the pump beam is $1600 \mathrm{~cm}^{-1}$ for Stokes multiphonon absorption and $1200 \mathrm{~cm}^{-1}$ for the anti-Stokes one, then the conditions to obtain avalanche up-conversion are completely fulfilled. Applying our calculations, $\beta$ is found to be $5 \times 10^{-6}$ and a clear avalanche behavior is observed (Fig. 3).

For $\mathrm{Nd}^{3+}$ in the same material, the pump being at $603.6 \mathrm{~nm}$ (only at $400 \mathrm{~cm}^{-1}$ from the ${ }^{2} H_{11 / 2}$ level) and assuming equal electronic absorption cross-sections, $\beta=3 \times 10^{-2}$ at room temperature. The corresponding curve in Fig. 2 is 
clearly in the CRESA domain. At low temperature, $\beta=1.7 \times 10^{-4}$ slightly above our limit, and an avalanche like behavior is observed.

\section{Avalanche pumped lasers}

In this section, we will analyse the advantage of avalanche pumping compared to a quasi-resonant scheme.

\subsection{Laser threshold and efficiency}

To optimize a laser action, a large level 3 population is required. Looking at Fig. $2, p_{3}^{\beta}$ is almost zero at the avalanche threshold. To obtain a laser emission with avalanche pumping, it is necessary to pump at rates much higher than the critical value $W_{c}$. So, an efficient a valanche pumped laser system requires a low avalanche threshold which will be obtained for a large cross-relaxation coefficient and a long lived intermediate state. The dependence of $p_{3}^{\beta}\left(W_{2}\right)$, represented in Figs. 2 and 3, shows clearly that the $p_{3}^{\beta}$ maximum values are obtain in the CRESA domain. As an example, we will consider the case of $\mathrm{Nd}^{3+}$ and $\mathrm{Er}^{3+}$ ions in $\mathrm{LiYF}_{4}$.

In the first case, $W_{\mathrm{c}}$ is quite high $\left(5800 \mathrm{~s}^{-1}\right)$ because of the relatively short lived level $2\left(\tau_{2}=500 \mu \mathrm{s}\right)$. As shown in Fig. 2 , at the pumping rate used to obtain a laser emission, the upper level population under resonant pumping is 2.7 times higher than the one obtained in the avalanche pumping scheme $\left(\beta=1.7 \times 10^{-4}\right)$. Even for $W_{2}$ pumping rates two times larger (corresponding to $200 \mathrm{KW} / \mathrm{cm}^{2}$ ) the ratio $p_{3}^{1} / p_{3}^{1.7 \times 10^{-4}}\left(W_{\mathrm{c}}\right)$ is still 1.7 (see Fig. 2).

In the case of $\mathrm{Er}^{3+}: \mathrm{LiYF}_{4}$, the threshold is very low $\left(140 \mathrm{~s}^{-1}\right)$, the parameters used in the calculations are those of Ref. [8]. This material can be then pumped easily at much higher pumping rate. To compare the gain obtained for the level 3 population. So, it is easy to compare both pumping schemes. For $W_{2} \gg W_{c}$, $p_{3}^{0}\left(W_{2}\right) / p_{3}^{1}\left(W_{2}\right) \approx 1-r_{31} / \alpha$ with $\alpha=50000 \mathrm{~s}^{-1}$ and $r_{31}=1250 \mathrm{~s}^{-1}$, Fig. 4 shows clearly that the upper level population is almost the same for both pumping schemes. As an example, for $W_{2}=2900 \mathrm{~s}^{-1}$ (corresponding to $250 \mathrm{KW} / \mathrm{cm}^{2}$ at $578 \mathrm{~nm}) p_{3}^{1} / p_{3}^{5 \times 10^{-6}}\left(W_{\mathrm{c}}\right)=1.003$.

Concerning the efficiency, resonant pumping always results in a higher level population but at the expense of the higher incident power if two excitation sources are used. For the systems with large cross-relaxation, resonant pumping will be less efficient than avalanche pumping. If we normalize $p_{3}^{\beta}$ by the total excitation power, i.e. $W_{3}(1+\beta)$, a valanche pumping in $\mathrm{Er}^{3+}: \mathrm{LiYF}_{4}$ is $28 \%$ more efficient than resonant one (Fig. 5). For other systems with moderate cross-relaxation, a valanche pumping will be less efficient. For $\mathrm{Nd}^{3+}: \mathrm{LiYF}_{4}$, the avalanche is $74 \%$ less efficient than the resonant excitation. On the other hand, CRESA (i.e. large values of $\beta$ ) will be more efficient than avalanche since, using a single beam, a strong ground state absorption is obtained without increasing substantially the pump power. From this result, we conclude that $\mathrm{Tm}^{3+}$ doped materials are probably the most efficient for nonresonant, single-source pumped up-conversion since they exhibit the smallest detuning between pump and ground state electronic absorption $[18,19]$.

In summary, avalanche systems are efficient only when pumped well above threshold. If this is not possible, resonant pumping will feed more efficiently level 3. 


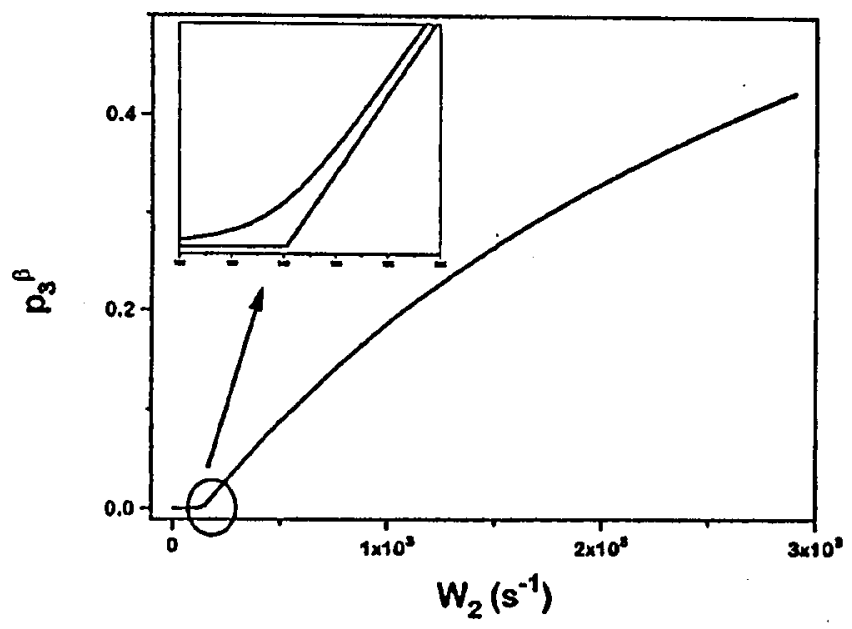

Fig. 4. Level 3 population dependence on $W_{2}$ pumping rate for $\mathrm{Er}^{3+}: \mathrm{YLiF}_{4}$. The curves $\beta=5 \times 10^{-6}$ and $\beta=1$ are very close except in the avalanche threshold region shown in the inset.

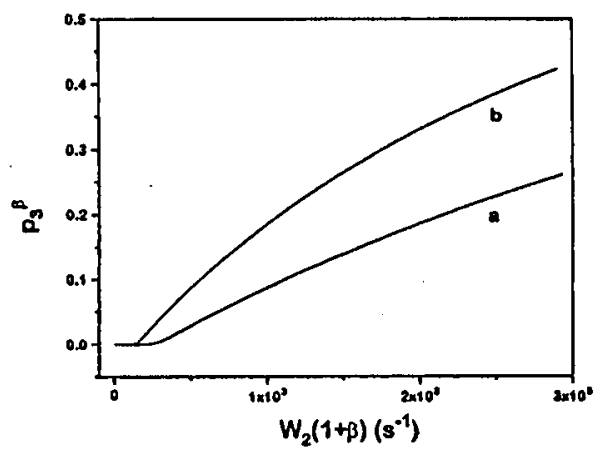

Fig. 5. Level 3 population on total pumping rate for $\mathrm{Er}^{3+}: \mathrm{YLiF}_{4} \cdot \beta=1$ (a), $\beta=$ $5 \times 10^{-6}(\mathrm{~b})$.

A good compromise between high level 3 population and low excitation power is found for single-beam pumped CRESA up-conversion.

\subsection{Stimulated emission}

To take into account an up-converted stimulated emission, its rate $q$ is introduced by substituting $r_{31}+q$ to $r_{31}$ in the rate equations since the laser is assumed to be a four-level one. This greatly simplifies the discussion: for example, the conclusions of the preceding section can be directly applied to the dependence of the output power $p_{3}^{\beta} q$ on $\beta$.

In Sec. 2.3.1 we pointed out that the relaxation of level 3 to level 1 was a critical parameter for an avalanche pumped system. This relaxation is of course in- 


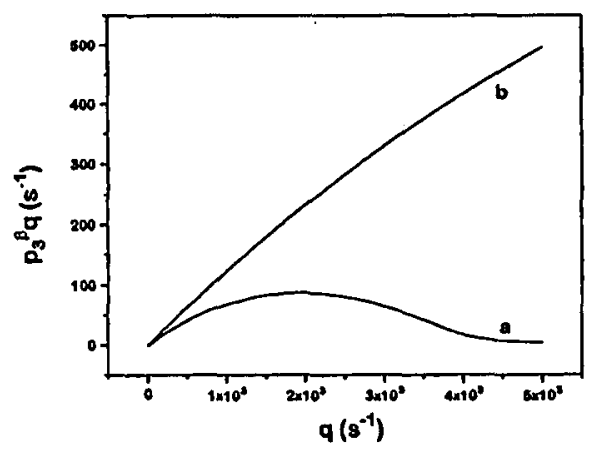

Fig. 6. Output power dependence on stimulated emission rate for $\mathrm{Nd}^{3+}: \mathrm{YLiF}_{4} \cdot \beta=$ $1.7 \times 10^{-4},(1+\beta) W_{2}=10000 \mathrm{~s}^{-1}(\mathrm{a}) ; \beta=1,(1+\beta) W_{2}=5000 \mathrm{~s}^{-1}$ (b).

creased by the up-converted laser emission, resulting in a higher threshold pumping rate $W_{\mathrm{c}}$ and a fast decrease in level 3 population.

As an example, $p_{3} q$ is plotted in Fig. 6 against $q$ for avalanche and resonant pumping of $\mathrm{Nd}^{3+}: \mathrm{LiYF}_{4}$. As expected, the output power of the avalanche laser is low and very sensitive to an increase in $q$. This result is in agreement with the very low value measured experimentally $(10 \mu \mathrm{W})$ [4]. Further agreement can be found in the observation by the same authors of a much stronger laser emission (but which is not up-converted) between level 3 and 2. In this case, of course, the strong $W_{2}$ pump between the same levels easily maintains a high population in level 3 even for a large stimulated emission. On the opposite, resonant pumping can sustain a high output power since level 2 and level 3 are efficiently populated. As in Sec. 3.1. a CRESA process obtained with a single pump beam will improve the maximal output power obtained with avalanche up-conversion without increasing the excitation pump power.

\section{Conclusion}

In the frame of a three-level model, a valanche up-conversion can be easily defined in the case of zero absorption from the ground state. It is possible to approach this limit for some rare earth doped materials, provided a large non-resonance between pump and ground state absorption or a low temperature. More precisely, a ratio between resonant and non-resonant pumping rates lower than $10^{-4}$ is necessary to observe a valanche up-conversion, provided that the fluorescence is properly scaled. Above this limit, the normalized up-converted emission does not show any pump threshold. Assuming multiphonon assisted absorption from the ground state, we showed that photon a valanche can occur in $\mathrm{Er}^{3+}$ doped $\mathrm{LiYF}_{4}($ at $300 \mathrm{~K})$ and $\mathrm{Nd}^{3+}: \mathrm{LiYF}_{4}$ (at low temperature) in agreement with experiments. Concerning laser action, avalanche pumping gives a lower population in the upper level than resonant excitation and therefore a higher laser threshold. Moreover, the avalanche up-conversion pumped lasers are very sensitive to stimulated emission from level 3 to 1 because of the very small pumping rate from level 1 to level 2 . This drawback is likely to seriously limit the output power and high temperature operation of 
such lasers. In opposition, the resonant pumping scheme, which allows an efficient feeding of levels 2 and 3 , can sustain a larger output power. However, a second pump beam, and therefore an increased total incident power, may be necessary for resonant excitation of the ground state. In this case, the most efficient design could be obtained with a single pump beam quite close to the electronic absorption, like in $\mathrm{Tm}^{3+}$-doped compounds.

\section{Acknowledgments}

The authors wish to thank Dr F. Auzel for helpful discussions and results available before publication.

\section{References}

[1] F. Auzel, IEEE 61, 758 (1973).

[2] J.S. Chivian, W.E. Case, D.D. Eden, Appl. Phys. Lett. 35, 124 (1979).

[3] M.E. Koch, A.W. Kueny, W.E. Case, Appl. Phys. Lett. 56, 1083 (1990).

[4] W. Lenth, R.M. Macfarlane, J. Lumin. 45, 346 (1990).

[5] T. Hebert, R. Wannemacher, R.M. Macfarlane, W. Lenth, Appl. Phys. Lett. 60, 2592 (1992).

[6] H. Ni, S.C. Rand, Opl. Lett. 16, 1424 (1991).

[7] B.P. Scott, F. Zhao, R.S.F. Chang, N. Djeu, Opt. Lett. 18, 113 (1993).

[8] F. Auzel, Y. Chen, J. Lumin. 65, 45 (1995).

[9] Y. Chen, F. Auzel, J. Phys. D, Appl. Phys. 27, 1 (1994).

[10] M.F. Joubert, S. Guy, B. Jacquier, Phys. Rev. B 48, 10031 (1993).

[11] E. Case, M.E. Koch, A.W. Kueny, J. Lumin. 45, 351 (1990).

[12] A.W. Kueny, W.E. Case, M.E. Koch, J. Opt. Soc. Am. B 10, 1834 (1993).

[13] F. Auzel, in: Radiationless Processes, Eds. B. Di Bartolo, V. Goldberg, Plenum, New York 1980, p. 250.

[14] N. Pelletier-Allard, R. Pelletier, Opt. Commun. 81, 247 (1991).

[15] F. Auzel, Phys. Rev. B 13, 2809 (1976).

[16] A. Brenier, L.C. Courrol, C. Pedrini, C. Madej, G. Boulon, Opt. Mater. 3, 25 (1994).

[17] A. Brenier, C. Garapon, C. Madej, C. Pédrini, G. Boulon, J. Lumin. 62, 147 (1994).

[18] A.A.S. da Gamma, G.F. de Sa, P. Porcher, P. Caro, J. Chem. Phys. 75, 2583 (1981).

[19] J.M. Dyson, S.M. Jaffe, H. Eilers, M.L. Jones, W.M. Dennis, M. Yen, J. Lumin. C0/61, 668 (1994). 